\title{
Caracterización del área de distribución geográfica potencial de las especies de aves psitácidas de la Península de Yucatán, México
}

\author{
Alexis Herminio Plasencia Vázquez \& Griselda Escalona Segura \\ El Colegio de la Frontera Sur (ECOSUR), Libramiento Carretero Campeche Km 1.5, Av. Rancho, Polígono 2-A, \\ Parque Industrial de Lerma, C.P. 24500. San Francisco de Campeche, Campeche, México; alexpla79@gmail.com, \\ gescalon@ecosur.mx
}

Recibido 20-II-2014. Corregido 14-VII-2014. Aceptado 13-VIII-2014.

\begin{abstract}
Characterization of the potential geographical distribution area of parrot species in Yucatan Peninsula, Mexico. Psittacidae family is one of the most endangered groups in Mexico, since many of their habitats are disappearing. In this research, we characterized the land cover of the potential geographical distribution area of eight extant parrot species within the Yucatan Peninsula. We used the Maximum Entropy algorithm (MaxEnt) and species historical records. To externally validate the models, we used presence and absence records from field observations (2010-2012). To characterize the distribution area, we used the vegetation and land use maps of INEGI Series IV (2007-2010). The models showed a good performance, according to the values of the area under the curve (AUC), which ranged between $0.88-0.95$ with the training data and between 0.82-0.91 with test data. We located most of the species in sites where the models predicted their presence. In the Peninsula, over $76 \%$ of the parrots' potential geographical distribution area is forested, except for Amazona oratrix. The subhumid tropical forest is the best represented, and the livestock for land use. The most affected species within the Peninsula are: Amazona farinosa and A. oratrix. The Calakmul Biosphere Reserve is the most important area for parrots' protection in the Peninsula. Knowing the characteristics of distribution areas is an essential part in the establishment of parrots' conservation strategies. Rev. Biol. Trop. 62 (4): 1509-1522. Epub 2014 December 01.
\end{abstract}

Key words: ecological niche models, habitat, parrots, Southeastern Mexico, threatened species.

La familia Psittacidae es muy diversa y es uno de los grupos biológicos más amenazados a nivel mundial (Forshaw, 2006). De las 22 especies de loros presentes en México (Juniper $\&$ Parr, 1998), solo una no aparece en alguna categoría de riesgo de la Norma Oficial Mexicana NOM-059-SEMARNAT-2010 (SEMARNAT, 2010) y ocho especies aparecen en el Apéndice I de la CITES (2013). Estas especies se encuentran bajo una fuerte presión por el tráfico ilegal para el mercado de mascotas, que en México se estima una tasa de captura de 65 000-78 500 ejemplares anualmente (Cantú, Sánchez, Grosselet, \& Silva, 2007) y por la pérdida de sus hábitats, que constituyen los principales factores que las afectan (MarínTogo et al., 2012).
En general, los psitácidos mexicanos se encuentran asociados a las selvas que suministran los recursos necesarios para su desarrollo (Collar, 1997), por lo que áreas sin perturbación humana son necesarias para el mantenimiento de sus poblaciones (Morales-Pérez, 2005). Para algunas especies de psitácidos las modificaciones antrópicas pueden resultar benéficas (ej. áreas agrícolas), al constituir una fuente de alimento más accesible (RomeroBalderas, Naranjo, Morales, \& Nigh, 2006). Sin embargo, los sitios de anidación están asociados generalmente a zonas con vegetación conservada y con presencia de árboles maduros (Morales-Pérez, 2005), por lo que la reducción de algunas poblaciones de loros se debe a la pérdida de un recurso clave como la cavidad 
para anidar, producida por la tala de los bosques (Berovides \& Cañizares, 2004). En México, se han utilizado los modelos de nicho ecológico para predecir la distribución geográfica potencial de diferentes especies de psitácidos y estimar cómo se ven afectadas por diferentes factores ambientales (Ríos-Muñoz \& NavarroSigüenza, 2009; Monterrubio-Rico, Renton, Ortega-Rodríguez, Pérez-Arteaga, \& CancinoMurillo, 2010; Rivera-Ortíz et al., 2013).

A nivel mundial las selvas tropicales desaparecen rápidamente debido a la deforestación y al cambio de uso del suelo, lo que implica una tendencia general de reemplazo de las selvas por áreas agrícolas y pastizales para ganado (Noss, Custi, \& Groom, 2006). México se encuentra entre los primeros lugares de países con mayor deforestación en el mundo y durante el periodo 2005-2010 la pérdida de las masas forestales fue de alrededor de 155 000ha por año (INEGI, 2014a). Para el 2007, se estimó que aproximadamente el $28 \%$ del total del territorio de México se encontraba cubierto por algún tipo de uso de suelo (INEGI, 2014b).

En la Península de Yucatán, las áreas de selva donde potencialmente se pueden localizar los psitácidos desaparecen rápidamente (Vester et al., 2007). Históricamente, el estado de Yucatán tuvo la mayor pérdida de cobertura vegetal por las actividades humanas (Daniels, Painter, $\&$ Southworth, 2008), aunque en los últimos años las selvas más afectadas han sido las que se encuentran al sur-occidente de Quintana Roo y sur-oriente de Campeche (Turner II, Geoghegan, \& Foster, 2004). Actualmente, el estado de Campeche tiene una tasa de deforestación de aproximadamente $31 \mathrm{ha} / \mathrm{año}$, el estado de Yucatán de 23ha/año, y Quintana Roo de 11ha/año (Céspedes-Flores \& Moreno-Sánchez, 2010). Si se mantienen estas tasas de deforestación, los hábitats de todas las especies de psitácidos presentes en la Península de Yucatán podrían verse seriamente afectados en los próximos años. En este contexto, el objetivo principal de este estudio es caracterizar la cobertura del suelo en el área de distribución geográfica potencial de los psitácidos presentes en la Península de Yucatán, como una medida del estado actual de sus hábitats. Esta información proporcionará las bases ecológicas sólidas para generar estrategias de conservación de los psitácidos y sus hábitats, que apoyen los esfuerzos de protección que se realizan para diferentes especies de loros en todo México.

\section{MATERIALES Y MÉTODOS}

Área de estudio: La investigación se realizó en la porción mexicana de la provincia biótica de la Península de Yucatán, que incluye los estados de Campeche, Quintana Roo y Yucatán, y se localiza al noreste de Centro América (Barrera, 1962).

Especies estudiadas y modelos de distribución: Se analizaron las ocho especies de psitácidos que habitan en la Península de Yucatán: perico de garganta oliva (Aratinga nana, A.na), loro frente blanca (Amazona albifrons, A.al), loro yucateco (Amazona xantholora, A.xa), loro frente roja (Amazona autumnalis, A.au), loro cabeza amarilla (Amazona oratrix, A.or), loro harinoso (Amazona farinosa, A.fa), loro corona blanca (Pionus senilis, P.se) y loro marrón encapuchado (Pyrilia haematotis, P.ha) (MacKinnon, 2005). Para A. xantholora y $A$. oratrix se utilizaron los mapas de distribución geográfica potencial obtenidos por PlasenciaVázquez, Escalona-Segura y Esparza-Olguín (2014). Para generar y validar los modelos de las seis especies restantes se usó la misma metodología propuesta por estos autores, así como las mismas fuentes de datos de los registros de presencia.

Los modelos se generaron con el uso de registros correspondientes al período 19102009, a partir de diferentes fuentes históricas: resultados de trabajo de campo que se obtuvieron de proyectos de investigación, consulta de bases de datos electrónicas (CONABIO: http://www.conabio.gob.mx/ y ORNISnet: http://www.ornisnet.org/) y revisión de literatura científica (Herrera-Herrera, 2001; GómezOrduño, 2006; Puc-Cabrera, 2008). Se usaron un total de 286 registros para $A$. nana, 256 para A. albifrons, 65 para A. autumnalis, 51 para $P$. 
senilis, 20 para $P$. haematotis y ocho para $A$. farinosa. Es importante resaltar que no se utilizaron coordenadas duplicadas para ninguno de los registros de presencia y todos eran espacialmente únicos, o sea cada uno estaba incluido en un pixel diferente. En la generación de los modelos de distribución geográfica potencial se usó el programa MaxEnt (Phillips, Anderson, \& Schapire, 2006) (http://www.cs.princeton. edu/ schapire/maxent/), que se considera como uno de los de mejor desempeño, y que tiene la ventaja de que se pueden obtener resultados relativamente adecuados con tamaños de muestra pequeños (Hernández, Graham, Master, \& Albert, 2006; Elith et al., 2011).

Como variables ambientales se utilizaron las capas derivadas de la interpolación de los datos de temperatura y precipitación desarrolladas por Cuervo-Robayo et al. (2013) y tres topográficas que se obtuvieron del proyecto Hydro-1K (http://gcmd.nasa.gov/records/ GCMD_HYDRO1k.html). Todas estas coberturas se re-muestrearon a una resolución de $0.008333^{\circ}$ (aproximadamente $1 \mathrm{~km}^{2}$ ), para ello se utilizó el programa ArcView 3.2 (ESRI, 1999). Para reducir el efecto de la colinealidad de las variables ambientales en los modelos, se realizó un análisis de correlación en la plataforma R 2.15.2, y se seleccionaron aquellas con valores de $|\mathrm{r}|<0.7$ (Dormann et al., 2012).

Para seleccionar entre los pares de variables con valores de $|r|>0.7$ se tuvo en cuenta que la variabilidad climática, determinada por las fluctuaciones durante los meses más extremos, puede limitar la reproducción de los loros, ya sea por un incremento en la depredación de los nidos o por disminuciones en la disponibilidad de alimento (Renton \& SalinasMelgoza, 2004). Finalmente, se seleccionaron ocho variables climáticas: temperatura máxima promedio del periodo más cálido $\left({ }^{\circ} \mathrm{C}\right)$, temperatura promedio del trimestre más seco $\left({ }^{\circ} \mathrm{C}\right)$, temperatura promedio del trimestre más cálido $\left({ }^{\circ} \mathrm{C}\right)$, temperatura promedio del trimestre más frío $\left({ }^{\circ} \mathrm{C}\right)$, precipitación del trimestre más lluvioso $(\mathrm{mm})$, precipitación del trimestre más seco $(\mathrm{mm})$, precipitación del trimestre más cálido $(\mathrm{mm})$, precipitación del trimestre más frío $(\mathrm{mm}), \mathrm{y}$ tres topográficas: modelo digital de elevación, pendiente e índice topográfico de humedad.

Durante la modelación se mantuvieron los parámetros predeterminados por el programa MaxEnt, como se ha sugerido por Phillips et al. (2006) y se ha validado en otros estudios (ej. Phillips \& Dudík, 2008). Se generaron 100 réplicas del modelo y se utilizó como método de remuestreo el de bootstrap. El 30\% de los registros de las especies se utilizó para la validación interna del modelo, menos en el caso de A. farinosa que al contar con pocos registros se usaron todos para generar el modelo. Se seleccionó como el mejor modelo el de la mediana. También se realizó una validación externa de los modelos, para la cual se utilizaron registros de presencia y ausencia de las especies, que se tomaron en 12 expediciones de campo realizadas en época seca y de lluvia durante 2011 y 2012, en diferentes áreas de la Península. Además, se usaron los registros recopilados del monitoreo realizado en el 2010 en la Reserva de la Biosfera Ría Lagartos y en diferentes áreas de Calakmul.

La toma de datos se realizó durante los recorridos que se llevaron a cabo desde el amanecer hasta aproximadamente las 10 de la mañana y por la tarde desde dos horas antes de la puesta de sol, que son los períodos de mayor actividad de los psitácidos (Gilardi \& Munn, 1998). Se tomaron las coordenadas de todos los loros detectados visual o auditivamente (Pizo \& Simao, 1997). En total, los registros de presencia y ausencia respectivos para cada especie fueron: A. nana (271 y 2), A. albifrons $(375 \mathrm{y}$ 2), A. autumnalis (45 y 33), P. senilis (33 y 32), P. haematotis (9 y 33) y A. farinosa (3 y 33).

Para obtener el mapa de presencia/ausencia se aplicaron cuatro valores de corte al mejor modelo de cada especie, para ello se tuvieron en cuenta cuatro algoritmos que se utilizan con frecuencia y se recomiendan en la literatura (Liu, Berry, Dawson, \& Pearson, 2005). Para determinar con cuál de los valores de corte que se utilizaron se obtenía el mejor mapa binario, se aplicó una matriz de confusión con los registros que se obtuvieron para validación 
en campo, que es un método que se utiliza para medir el desempeño de los modelos (Fielding $\&$ Bell, 1997). Con esta matriz, se determinó la coincidencia entre los registros de validación externa y la predicción que hace el modelo sobre el espacio geográfico.

\section{Caracterización del área de distribu-}

ción: Para caracterizar el área de distribución geográfica potencial de las ocho especies de psitácidos se utilizaron 15 archivos digitales de la carta de uso de suelo y vegetación correspondientes a la Serie IV de INEGI (2010), que en su conjunto abarcan la Península de Yucatán y son las más actuales para México. El mapa de cada especie se superpuso sobre la Serie IV y se calculó el área que representaba cada uso del suelo y cada tipo de vegetación dentro del área de distribución geográfica potencial; para ello se utilizó el programa ArcView 3.2 (ESRI, 1999). Además, se calculó el porcentaje del área de distribución geográfica potencial de cada una de las especies de psitácidos que estaba contenida dentro de las áreas naturales protegidas existentes en la Península de Yucatán (http://www.conanp.gob.mx).

\section{RESULTADOS}

Los modelos de consenso (mediana) para las seis especies tuvieron un buen desempeño y alcanzaron valores del AUC entre 0.880.95 con los datos de entrenamiento, y entre 0.82-0.91 con los datos de prueba. En general, los mejores mapas binarios de distribución geográfica potencial se obtuvieron con el algoritmo del valor mínimo en un punto de entrenamiento (minimum training presence). Con los demás algoritmos, se observó una mayor subestimación del área de distribución de los psitácidos en la Península de Yucatán (Cuadro 1). Los mapas binarios de distribución geográfica potencial de las seis especies de loros en la Península de Yucatán se muestran en la figura 1.

De manera general, durante los muestreos de validación en campo se localizó a la mayoría de las especies en los sitios donde los modelos predijeron presencia. En total para validación externa se visitaron 20 áreas entre 2011 y 2012, con un total de 276 horas de muestreo. Además, se incluyeron los registros de muestreos realizados en el 2010 en las Reservas de la Biosfera Ría Lagartos, Yucatán y Calakmul, Campeche. El mayor esfuerzo de muestreo se concentró en la porción sur o base de la Península de Yucatán, que es donde se localizó el mayor número de especies. Las áreas que se visitaron para validación externa de los modelos, con el estado al que pertenecen y el total de horas de esfuerzo de muestreo (mostradas entre paréntesis), fueron: localidad El Tablón,

\section{CUADRO 1}

Valores de corte evaluados con cuatro algoritmos para obtener los modelos de distribución geográfica potencial de seis de las especies de psitácidos presentes en la Península de Yucatán

TABLE 1

Thresholds evaluated using four algorithms to obtain the potential geographical distribution models of six from the extant parrot species within the Yucatan Peninsula

\begin{tabular}{lcccccc}
\multicolumn{1}{c}{ Algoritmo } & A. albifrons & A. nana & A. autumnalis & P. haematotis & P. senilis & A. farinosa \\
MTP (\%) & 4 & 8 & 5 & 22 & 7 & 33 \\
10P (\%) & 27 & 28 & 22 & 35 & 26 & 33 \\
OC (\%) & 36 & 40 & 28 & 40 & 29 & 43 \\
Max (\%) & 38 & 42 & 28 & 38 & 27 & 47 \\
\hline
\end{tabular}

MTP: presencia mínima de los puntos de entrenamiento, 10P: 10 percentil, OC: sensibilidad y especificidad equivalentes y Max: sensibilidad máxima de entrenamiento más especificidad.

MTP: minimum training presence, 10P: 10 percentile, OC: equal training sensitivity and specificity and Max: maximum training sensitivity plus specificity. 

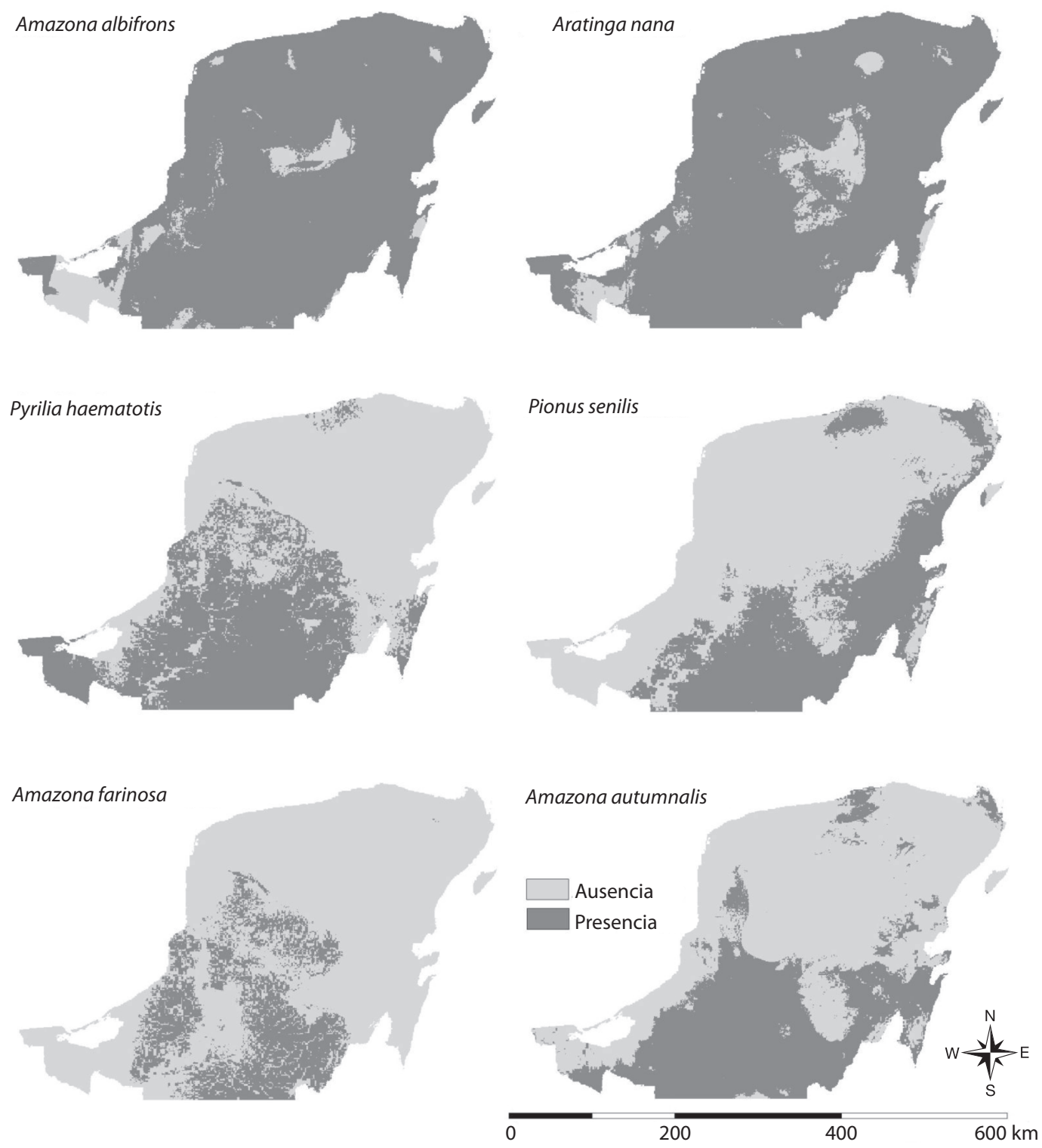

Fig. 1. Distribuciones geográficas potenciales de A. albifrons, A. nana, P. haematotis, P. senilis, A. farinosa y A. autumnalis en la Península de Yucatán, México obtenidas con el algoritmo de Máxima Entropía (MaxEnt).

Fig. 1. Potential geographical distributions of A. albifrons, A. nana, P. haematotis, P. senilis, A. farinosa and A. autumnalis within the Yucatan Peninsula, Mexico, obtained with the Maximum Entropy algorithm (MaxEnt).

Campeche (20); Isla de Cozumel, Quintana Roo (30); Reserva de la Biosfera Sian Ka'an, Quintana Roo (30); Reserva de la Biosfera Los Petenes, Campeche (24); sitio arqueológico Edzná, Campeche (4); Área de Protección de Flora y Fauna Otoch Ma'ax Yetel Kooh,
Yucatán-Quintana Roo (24); Palizada, Campeche (20); Atasta, Campeche (6); localidad Tigre Grande, Yucatán (18); Laguna de Términos, Campeche (12); Laguna de Atasta, Campeche (12); Bahía de Chetumal, Quintana Roo (16); Xcalak, Quintana Roo (8); Mahahual, Quintana 
Roo (10); localidad Dos Lagunas, Campeche (6); localidad Arroyo Negro, Campeche (6); localidad Tres Garantías, Quintana Roo (12); Laguna Muchucux, Quintana Roo (6); localidad Pantera, Quintana Roo (6) y localidad Divorciados, Quintana Roo (6).

Solo en el caso de P. haematotis y A. farinosa se dificultó su localización. Esta última especie no se detectó en ninguna de las áreas visitadas, a pesar de ser sitios donde históricamente se había reportado, o que cumplen con las características del hábitat adecuadas según lo que se conoce sobre la historia natural de la especie. Solo se obtuvieron registros de avistamientos de $A$. farinosa facilitados por uno de los guías de campo que apoyó durante los recorridos en áreas de la localidad Tres Garantías en Quintana Roo (18'4'37'7" N - 896'29"3" O).

Amazona albifrons es la especie que ocupó el mayor área de distribución geográfica potencial dentro de la Península de Yucatán: $127190.0 \mathrm{~km}^{2}$, que representó el $92.1 \%$ del área total de la Península. Le siguió $A$. nana con $126223.3 \mathrm{~km}^{2}$, que representó el $91.4 \%$ de ocupación del área total, y en tercera posición estuvo $A$. xantholora con $98982.9 \mathrm{~km}^{2}$ (71.7\%). Para P. haematotis el área de distribución geográfica potencial fue de $56650.3 \mathrm{~km}^{2}$ (41.0\%), mientras que la de A. autumnalis de $53270.4 \mathrm{~km}^{2}(38.6 \%)$, y la de $P$. senilis 48 $136.5 \mathrm{~km}^{2}(34.9 \%)$. Las especies con menor área de distribución geográfica potencial fueron: A. farinosa con $29480.3 \mathrm{~km}^{2}(21.4 \%)$ y $A$. oratrix $5504.9 \mathrm{~km}^{2}(4.0 \%)$.

De manera general, más del $76 \%$ del área de distribución geográfica potencial de cada uno de los psitácidos en la Península estaba ocupada por selva (se incluyen los manglares, bosque de encino, palmares y peten), excepto en el modelo de $A$. oratrix, donde el $63.5 \%$ del área estaba ocupada por diferentes usos de suelo y otros tipos de vegetación (Cuadro 2). Resulta necesario señalar que los petenes se han descrito como pequeñas "islas" de vegetación arbórea, principalmente de selva mediana subperennifolia, que se encuentran inmersas en medio de amplias zonas inundables dominadas por pastizales y manglares de poca altura
(Barrera, 1982). En proporción al área de distribución geográfica potencial, las especies que contaron con un mayor porcentaje de cobertura boscosa fueron: $A$. farinosa $(84.7 \%)$ y $P$. senilis (81.7\%). Para A. xantholora, el 79.4\% del área de distribución geográfica potencial fue selva, mientras que para $P$. haematotis fue $78.2 \%$, para A. autumnalis $78.1 \%$, A. albifrons $77.9 \%$ y $A$. nana $76.8 \%$. La selva mediana subperennifolia fue la mejor representada dentro de las áreas de distribución potencial de los psitácidos en la Península, mientras que de los usos de suelo, el que ocupó una mayor proporción fue el pecuario, seguido por los cultivos agrícolas (Cuadro 2).

En cuanto a las áreas de distribución potencial dentro de las áreas naturales protegidas, para A. albifrons, $14506.0 \mathrm{~km}^{2}$ se encontraron dentro de algún área protegida, lo cual representó el $11.4 \%$ de su distribución potencial en toda la Península de Yucatán. En el caso de Aratinga nana, $15673.8 \mathrm{~km}^{2}$ (12.4\%) de su distribución potencial formaron parte de áreas protegidas, mientras que para $A$. xantholora fueron $11390.9 \mathrm{~km}^{2}(11.5 \%)$. Para A. autumnalis, $9440.5 \mathrm{~km}^{2}(17.7 \%)$ de su distribución potencial estuvieron incluidos en las áreas protegidas, mientras que para $P$. haematotis estuvieron incluidos $10700.4 \mathrm{~km}^{2}(18.9 \%) \mathrm{y}$ para P. senilis $11364.0 \mathrm{~km}^{2}(23.6 \%)$.

La distribución potencial de $A$. oratrix y $A$. farinosa solo estuvo representada dentro de un área protegida en la Península de Yucatán. En el caso de $A$. oratrix, solo $2748.6 \mathrm{~km}^{2}$ (49.9\%) de su área total de distribución geográfica potencial se encontró dentro del Área de Protección de Flora y Fauna Laguna de Términos, mientras que para $A$. farinosa, $5328.8 \mathrm{~km}^{2}$ (18.1\%) del total de su área de distribución potencial estuvieron incluidos en la Reserva de la Biosfera de Calakmul. Las demás especies aparecieron distribuidas en diferentes áreas protegidas. Sin embargo, la más importante para todas fue la Reserva de la Biosfera de Calakmul, ya que cubría más del $44 \%$ de la distribución potencial en relación al total de áreas protegidas. 
$\frac{0}{0}$

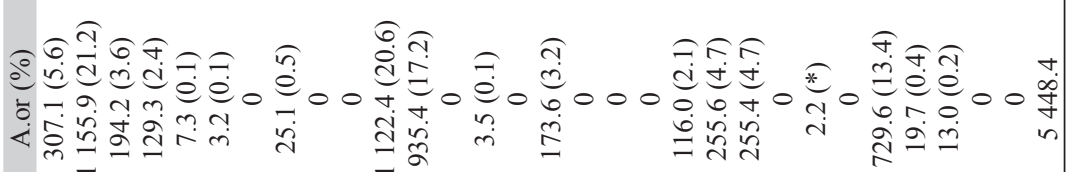

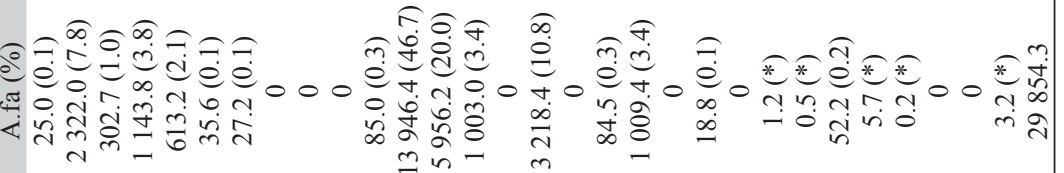

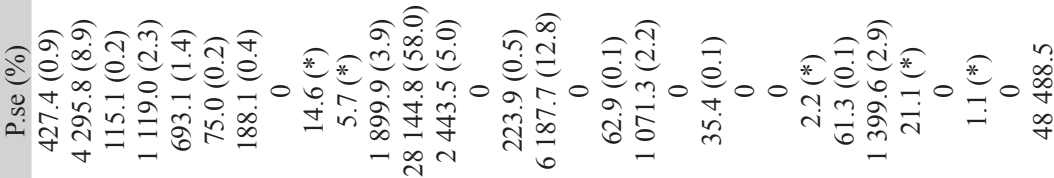

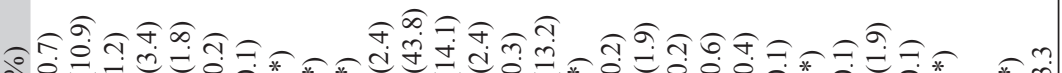

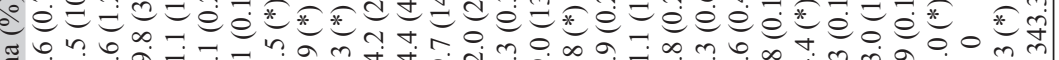

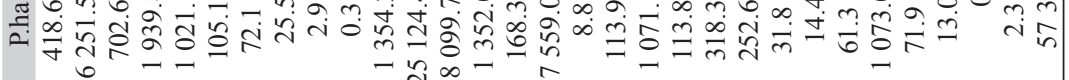

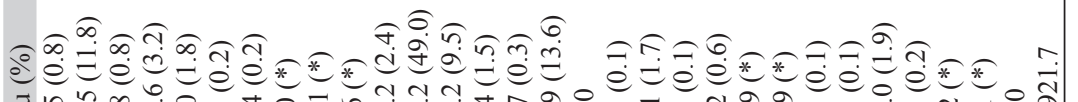

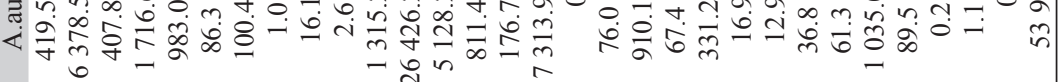

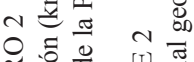

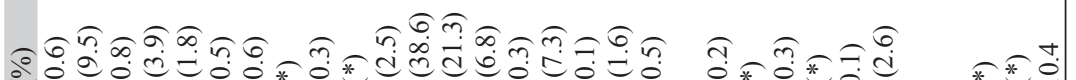

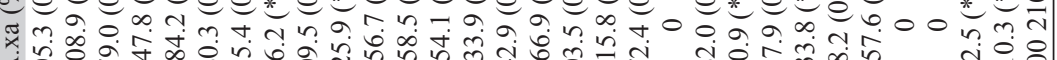

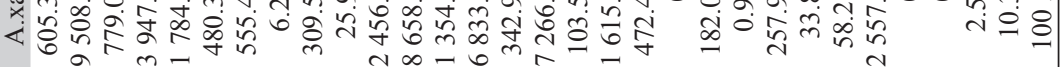

o

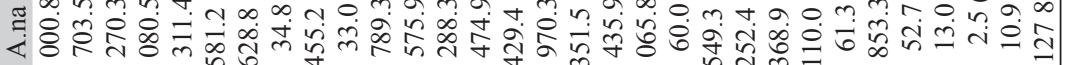

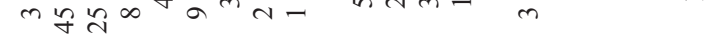

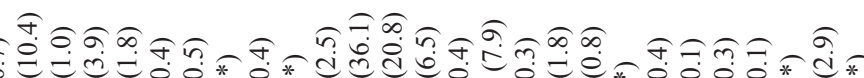

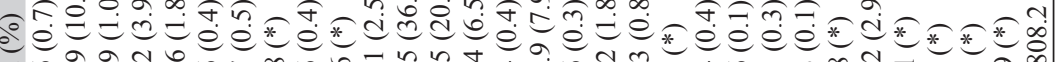

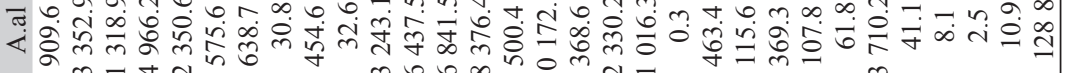

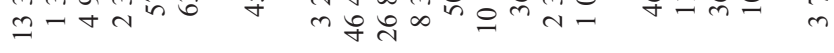

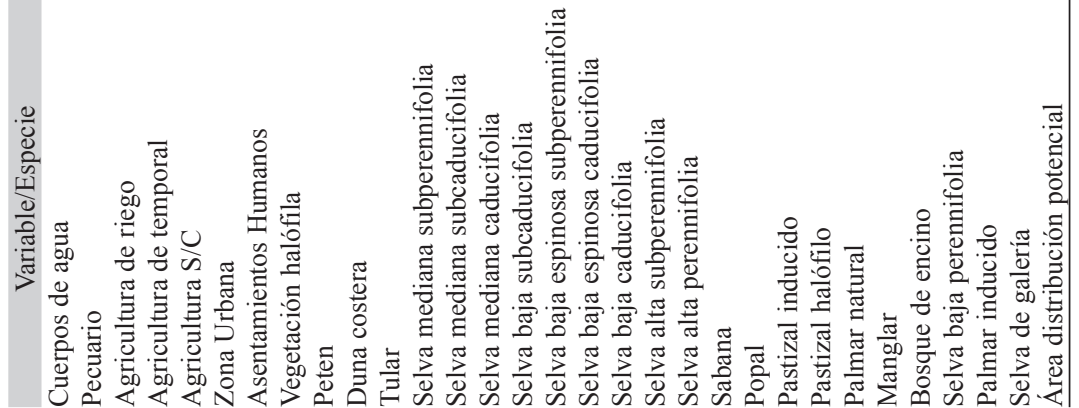




\section{DISCUSIÓN}

Los modelos de distribución geográfica potencial que se obtuvieron para los loros en la Península de Yucatán fueron más detallados y tuvieron una base ecológica y matemática mejor definida que las representaciones poligonales que se encontraron en las guías de campo (e.g. Howell \& Webb, 1995; Forshaw, 2006). Las áreas de presencia que se predijeron en este estudio no coincidieron exactamente con los modelos que se obtuvieron con las mismas especies de psitácidos para todo México según Ríos-Muñoz \& Navarro-Sigüenza (2009). Estos autores solo utilizaron datos de colecciones, mientras que en este estudio también se incluyeron datos de campo. Además, para este estudio se recopiló una mayor cantidad de registros de presencia para algunas de las especies, lo cual permitió obtener modelos estadísticamente más robustos (Wisz et al., 2008). También, se comprobó que MaxEnt funciona bien para predecir zonas de presencia en sitios donde no hay datos de muestreo o para especies de las cuales se cuenta con escasos registros de presencia (Hernández et al., 2006).

En la mayor parte del sureste mexicano las poblaciones de A. macao, $P$. haematotis, $P$. senilis, $A$. autumnalis, $A$. farinosa y $A$. oratrix se han visto seriamente afectadas por los cambios de uso de suelo y por la pérdida de gran parte de las selvas altas perennifolias, las cuales han constituido uno de los principales hábitats para estas especies (Ríos-Muñoz \& NavarroSigüenza, 2009). En general, en la Península de Yucatán los diferentes usos de suelo no ocuparon áreas continuas, y se encontraron dispersos en la mayor parte del territorio. Sin embargo, los diferentes tipos de vegetación presentes en la Península se fragmentaron gradualmente, lo cual conllevó a que se facilitara el acceso de los humanos y otras especies a algunas áreas que históricamente permanecieron aisladas e inaccesibles.

Si se tiene en cuenta la información que publicó el INEGI (2010), en la Península de Yucatán la selva alta perennifolia y la selva alta subperennifolia estuvieron representadas en muy baja proporción, por lo que no constituyeron hábitats que aportaran significativamente al mantenimiento de las poblaciones de loros en el área. Los principales remanentes de selva alta perennifolia que persisten en la Península se localizaron en la porción sur; que incluyó las zonas con mayor actividad ganadera dentro del estado de Campeche, por lo que se ha perdido gran parte de la cobertura vegetal (VillalobosZapata \& Mendoza Vega, 2010).

La selva mediana subperennifolia fue la más abundante en toda la Península de Yucatán al cubrir aproximadamente el $35 \%$ del territorio y fue la formación vegetal más importante en las áreas de distribución potencial de las ocho especies de psitácidos. En todo México, este tipo de vegetación representó aproximadamente el 18\% del área total de selvas (INEGI, 2010) y la Península es una de las áreas donde estuvo mejor representada. En la Península este tipo de selva solo se encontró protegida dentro de la Reserva de la Biosfera de Calakmul en Campeche, por lo que se hace necesario establecer estrategias de conservación a lo largo del estado de Quintana Roo, donde está totalmente desprotegida, y en los municipios Carmen y Palizada en Campeche, donde ha sido seriamente fragmentada por la actividad maderera y por los usos pecuarios (Turner II et al., 2004). Estas estrategias se deben enfocar en la identificación de las empresas y los grupos comerciales que impulsan la actividad maderera en la región, hacia los cuales se deben dirigir campañas de presión pública; que a su vez deben estar respaldadas por campañas educacionales para los consumidores (Butler \& Laurance, 2008). La selva mediana subcaducifolia fue la segunda más importante para la mayoría de los loros de la Península y solo el 3\% de la extensión que ocupa en la región se encuentra protegida dentro de las Reservas de la Biosfera de Calakmul y Ría Lagartos y el Área de Protección de Flora y Fauna Yum Balam, por lo que los mismos esfuerzos y estrategias de conservación se deben enfocar con prioridad en esta formación vegetal.

Aunque se comprobó que han existido áreas extensas de selvas en la Península, hay 
que considerar que toda esta zona estuvo influenciada por las actividades de los Mayas en la etapa prehispánica y por los nuevos pobladores en los últimos dos siglos (Martínez \& Galindo Leal, 2002). La recuperación de las áreas que sufrieron transformaciones o de aquellas que se han vuelto a regenerar, no significa que las poblaciones de las demás especies que allí se distribuían se hayan recuperado, ya que pudo ocurrir un proceso de defaunación (Redford, 1992). Por lo tanto, se requiere complementar las estimaciones actuales de distribución de las especies de psitácidos con datos de campo precisos, con el fin de evaluar el estado actual de sus poblaciones y desarrollar estrategias de conservación geográficamente apropiadas (Marín-Togo et al., 2012). Los mayores esfuerzos se deben dirigir hacia la porción sur de la Península, que albergó a las ocho especies de loros, como se pudo constatar durante los muestreos para validación externa de los modelos. Se deben realizar conteos para estimar la abundancia de los psitácidos en esta zona y así contar con elementos integrales para decidir cuáles serán los sitios prioritarios de conservación.

En relación a los diferentes usos de suelo en la Península, se observó que las actividades agropecuarias ocuparon la mayor cantidad de territorio. Sin embargo, las áreas de cultivo se incrementan cada día más y en los sitios donde se desarrollan se ha favorecido la entrada de cazadores furtivos y de personas que se dedican a la tala ilegal. Para el 2012, en el estado de Campeche la superficie que se cultivó con pastos ocupó la mayor área con un total de $3955.9 \mathrm{~km}^{2}$, seguida por el cultivo de maíz con $1864.6 \mathrm{~km}^{2}$, mientras que en Quintana Roo prevaleció el cultivo de caña de azúcar $\left(248.9 \mathrm{~km}^{2}\right)$ y en Yucatán los pastos $\left(2401.2 \mathrm{~km}^{2}\right)$ (INEGI, 2014c).

Se infirió que los cambios de usos del suelo tendrían un efecto mucho mayor sobre las especies que habitan en los trópicos, ya que tienden a tener rangos de distribución más restringidos que las hacen más vulnerables a la extinción (Jetz, Wilcove, \& Dobson, 2007). Sin embargo, muchas especies de psitácidos han demostrado ser bastante resistentes a los cambios e incluso se han convertido en plagas dentro y fuera de sus rangos de distribución históricos (Bucher, 1992; Cepeda-González, 2012). En la Península se ha documentado y se observó durante los muestreos en campo, que algunas especies como A. albifrons, A. xantholora y $A$. autumnalis pueden utilizar las áreas pecuarias y agrícolas para alimentarse y ocasionalmente para anidar, siempre que los árboles adecuados estén presentes en estas áreas o el hábitat primario esté disponible en áreas cercanas (Galindo-Leal, 1999). La capacidad de adaptación de estos loros a los cambios pareciera resultar positiva para garantizar la perdurabilidad de las especies, pero el uso de áreas más expuestas a la presencia humana puede hacerlas más vulnerables al saqueo ilegal.

El panorama más crítico en la Península se encontró para $A$. farinosa y A. oratrix. En el caso de $A$. farinosa, su ausencia durante los muestreos de validación y el número reducido de registros históricos de la especie sugirieron que sus poblaciones no son abundantes en la Península o que no ha sido un residente permanente. Además, los testimonios e información que brindaron los guías de campo apuntaron a que la especie solo aparece en determinadas áreas durante la etapa no reproductiva (generalmente entre los meses de julio-septiembre) y la han observado moverse desde la frontera con Guatemala hacia las áreas de la localidad Tres Garantías en Quintana Roo. Durante la visita que se realizó en el mes de septiembre de 2012 a la localidad Tres Garantías no se observó la especie, pero no se contó con el tiempo suficiente para realizar una búsqueda intensiva. En el caso de especies tan difíciles de localizar se tienen que planificar visitas al campo con mayor periodicidad, se deben priorizar aquellos meses en los que haya evidencia de un incremento en la probabilidad de ocurrencia. Con estas evidencias se pudo inferir que $A$. farinosa puede realizar migraciones desde El Peten en Guatemala, lo cual se comprobó solo en una ocasión según un reporte de Bjork (2004).

También se consideró que, de acuerdo al conocimiento de la ecología de A. farinosa, 
esta especie solo se alimenta en la selva perennifolia primaria, principalmente en el dosel de los árboles y desaparece de las áreas perturbadas pues estas condiciones no satisfacen sus requerimientos ecológicos (Ridgely, 1981). Dentro de su área de distribución potencial las selvas perennifolias estuvieron ausentes y al parecer la especie solo encontró condiciones favorables para alimentarse en un grupo limitado de sitios relativamente conservados, entre ellos la localidad Tres Garantías en Quintana Roo o en algunas zonas dentro de la Reserva de la Biosfera de Calakmul.

En el caso de A. oratrix, el área de distribución geográfica potencial que ocupó dentro de la Península es muy limitada y solo menos de la mitad mantuvo la cobertura de selvas necesaria para su desarrollo. Esta especie ha sido documentada principalmente en áreas de selva primaria y requiere de árboles altos y maduros para anidar, por lo que generalmente no puede adaptarse a hacerlo en áreas modificadas (Renton, 2002). La evaluación rápida realizada por Macías Caballero e Iñigo Elías (2003) mostró que esta especie presenta uno de los valores más altos de abundancia relativa en la Península de Yucatán. Sin embargo, en el área que ocupó potencialmente la especie en la Península están ausentes las selvas subperennifolias conservadas que le proporcionan un hábitat óptimo para su reproducción (MonterrubioRico et al., 2010), lo cual impide que este loro pueda reproducirse adecuadamente y mantener una población viable.

Los valores de abundancia relativa elevados pueden ser engañosos, ya que pueden solo ser un producto de la elevada longevidad del grupo (según Munshi-South y Wilkinson (2006), el promedio de vida del género Amazo$n a$ es de 33.5 años), a pesar de que las tasas de natalidad puedan ser muy bajas. A la par de los estudios de abundancia y si las características de cada especie lo permite, se deben determinar los grupos de edades a los cuales pertenecen los individuos que se detecten en campo. Además, aunque la especie pueda encontrar las condiciones adecuadas para reproducirse, la actividad ganadera ha modificado mucho esta área $\mathrm{y}$ solo predominan los fragmentos de vegetación dentro de las extensas áreas de potreros (Villalobos-Zapata \& Mendoza Vega, 2010), lo cual facilita el acceso a los nidos y puede aumentar la vulnerabilidad de los pollos ante la depredación. Desafortunadamente, aunque Macías Caballero e Iñigo Elías (2003) mostraron evidencias sobre la importancia de la población de A. oratrix en la Península de Yucatán, los principales esfuerzos para el estudio de la especie se han enfocado en las poblaciones del Pacífico (Monterrubio-Rico et al., 2007; Téllez García, 2008; Monterrubio-Rico et al., 2010) y en la parte superior del Golfo de México (EnkerlinHoeflich, 1995).

En relación a las áreas naturales protegidas presentes en la Península de Yucatán, para la mayoría de los psitácidos estas cubrieron menos del $50 \%$ de sus áreas de distribución potencial, lo cual pone en duda la efectividad que estas puedan tener en la protección de estas especies. La Reserva de la Biosfera Calakmul fue la de mayor importancia para los loros en la Península por su extensión y por el número de especies que alberga. Sin embargo, por el área tan grande que ocupa los sistemas de vigilancia y protección no son completamente efectivos, ya que no cuentan con el personal ni los recursos suficientes para abarcar toda la zona. Sin duda, decretar nuevas áreas naturales protegidas dentro de la Península sería una buena estrategia para la protección de los psitácidos, pero primero se debe garantizar que en las existentes los planes de manejo funcionen adecuadamente. Actualmente se deben fortalecer los mecanismos de vigilancia dentro de las áreas naturales protegidas y se deben desarrollar campañas educativas que involucren a los pobladores que tienen más contacto con éstas. Además, se deben establecer mecanismos que incentiven a las personas a desarrollar más actividades que involucren el pago por servicios ambientales o buscar nuevas vías de remuneración a partir de las acciones de conservación, para evitar así que la captura de loros para el tráfico ilegal sea una vía de subsistencia dentro de algunas comunidades rurales. 
Es necesario destacar que el conocimiento de las características de las áreas de distribución de las especies, a partir de la utilización de modelos ecológicos de nicho, puede ser parte fundamental para establecer estrategias de conservación de los psitácidos. Existen otros factores que no fueron evaluados en este estudio y que pueden ser muy útiles en la caracterización de las áreas de distribución de los loros, como son los diferentes niveles de disturbio del hábitat y la fragmentación forestal (Jetz et al., 2007; Ríos-Muñoz \& Navarro-Sigüenza, 2009). Además, se debe tener información más detallada de las afectaciones de las poblaciones por el cambio de uso de suelo. También, se deben hacer estimaciones del impacto que puede tener el cambio climático global sobre las poblaciones de loros en la Península, dada su ubicación geográfica. Sin embargo, actualmente la protección de las áreas identificadas como prioritarias para la conservación de los psitácidos dentro de la Península debe ser la tarea principal. De igual manera, se deben llevar a cabo acciones de vigilancia en las zonas más vulnerables para evitar que se incremente la captura ilegal para el mercado de mascotas.

\section{AGRADECIMIENTOS}

A José del Carmen Puc, María Cristel Aguirre, Eloy Victoria Chan, Gilberto Quintal, Mario L. Palomo y a todas las personas que brindaron sus servicios como guías en el trabajo de campo. Al personal de las áreas protegidas: Reservas de la Biosfera Sian Ka'an y Los Petenes, Parque Nacional Arrecifes de Cozumel, Fundación de Parques y Museos de Cozumel, Reserva de la Biosfera de los Petenes, Áreas de Protección de Flora y Fauna Otoch Ma'ax Yetel Kooh y Laguna de Términos. A los habitantes de las localidades El Tablón, Arroyo Negro, Dos Lagunas, Divorciados, Pantera y Tigre Grande. A todos los que facilitaron registros de los loros y pericos en la Península de Yucatán: Barbara MacKinnon, Adolfo Navarro-Sigüenza, Cristóbal Cáceres, Javier Salgado Ortiz, Claudia Macías y Jesús Vargas Soriano. Al INEGI por facilitar las coberturas de Uso de Suelo y Vegetación. A Yarelys Ferrer-Sánchez, Katherine Renton, Ligia Guadalupe Esparza, Dennis Denis Ávila Octavio R. Rojas Soto, Enrique Martínez Meyer, César. A. Ríos Muñoz y A. Townsend Peterson por sus comentarios. Al CONACyT por el apoyo económico brindado a través de la beca 239499 y 21467. A los revisores del manuscrito por sus sugerencias y comentarios.

\section{RESUMEN}

La familia Psittacidae es una de las más amenazadas en México y los hábitats donde se distribuyen desaparecen. En este estudio se caracterizó la cobertura del suelo del área de distribución geográfica potencial de las ocho especies de psitácidos presentes en la Península de Yucatán. Se utilizó el algoritmo de Máxima Entropía (MaxEnt) y registros históricos de las especies. Para validar externamente los modelos se utilizaron registros de presencia y ausencia tomados en campo (2010-2012). Para caracterizar el área de distribución se utilizó la carta de uso de suelo y vegetación Serie IV de INEGI (2007-2010). Los modelos tuvieron un buen desempeño, de acuerdo a los valores del área bajo la curva (AUC), que oscilaron entre 0.88-0.95 con los datos de entrenamiento y entre $0.82-0.91$ con los de prueba. Se localizó a la mayoría de las especies en los sitios donde los modelos predecían presencia. Más del 76\% del área de distribución geográfica potencial de los psitácidos en la Península está ocupada por selva, excepto para $\mathrm{Ama-}$ zona oratrix. La selva mediana subperennifolia es la mejor representada en las áreas de distribución y de los usos de suelo el mejor representado es el pecuario. La especies más afectadas dentro de la Península son: Amazona farinosa y A. oratrix. La Reserva de la Biosfera de Calakmul es la más importante para la protección de los psitácidos en la Península. El conocimiento de las características de las áreas de distribución es parte fundamental en el establecimiento de estrategias de conservación de los psitácidos.

Palabras clave: especies amenazadas, hábitat, modelos de nicho ecológico, loros, sureste mexicano.

\section{REFERENCIAS}

Barrera, A. (1962). La Península de Yucatán como provincia biótica. Revista de la Sociedad Mexicana de Historia Natural, 23, 71-105.

Barrera, A. (1982). Los petenes del noroeste de Campeche. Su exploración ecológica en perspectiva. Biótica, 7, 163-169. 
Berovides, V. \& Cañizares, M. (2004). Diagnóstico del decline de los psitácidos cubanos y su posible solución. Biología, 18(2), 109-112.

Bjork, R. D. (2004). Delineating pattern and process in tropical lowlands: mealy parrot migration dynamics as a guide for regional conservation planning (Doctoral thesis). Oregon State University, Corvallis, Oregon, USA.

Bucher, E. (1992). Neotropical parrots as agricultural pests. In S. R. Beissinger \& N. F. R. Snyder (Eds.), New World parrots in crisis. Solutions from conservation biology (pp. 201-219). New York: Smithsonian Inst. Press.

Butler, R. A. \& Laurance, W. F. (2008). New strategies for conserving tropical forests. Trends in Ecology \& Evolution, 23(9), 469-472.

Cantú, G. J. C., Sánchez, M. E., Grosselet, M., \& Silva, J. (2007). Tráfico ilegal de pericos en México. Una evaluación detallada. México: Defenders of Wildlife.

Cepeda-González, M. F. (2012). Monitoreo para conservación: ensamble de aves de la Reserva de la Biosfera Ría Lagartos (Tesis de doctorado). El Colegio de la Frontera Sur, Lerma, Campeche, México.

Céspedes-Flores, S. E. \& Moreno-Sánchez, E. (2010). Estimación del valor de la pérdida de recurso forestal y su relación con la reforestación en las entidades federativas de México. Investigación ambiental, 2(2), 5-13.

CITES (2013). Convention on international trade in endangered species of wild fauna and flora. Appendices I, II and III. International Union for Conservation of Nature IUCN. Recuperado de http://www. cites.org/sites/default/files/eng/app/2013/E-Appendices-2013-06-12.pdf

Collar, N. J. (1997). Family Psittacidae (Parrots). In J. Del Hoyo, A. Elliot, \& J. Sargatal (Eds.), Handbook of the birds of the World (Vol. 4, pp. 280-477). Barcelona: Lynx Edicions.

Cuervo-Robayo, A. P., Téllez-Valdés, O., Gómez, M., Venegas-Barrera, C., Manjarrez, J., \& MartínezMeyer, E. (2013). An update of high-resolution monthly climate surfaces for Mexico. International Journal of Climatology. Recuperado de http://onlinelibrary.wiley.com/doi/10.1002/joc.3848/pdf

Daniels, A., Painter, K., \& Southworth, J. (2008). Milpa imprint on the tropical dry forest landscape in Yucatan, Mexico: Remote sensing and field measurement of edge vegetation. Agriculture, Ecosystems and Environment, 123(4), 293-304.

Dormann, C. F., Elith, J., Bacher, S., Buchmann, C., Carl, G., Carré, G., \& Lautenbach, S. (2012). Collinearity: a review of methods to deal with it and a simulation study evaluating their performance. Ecography, 35, 001-020.
Elith, J., Phillips, S. J., Hastie, T., Dudík, M., Chee, Y. E., \& Yates, C. J. (2011). A statistical explanation of MaxEnt for ecologists. Diversity and Distributions, 17, 43-57.

Enkerlin-Hoeflich, E. C. (1995). Comparative ecology and reproductive biology of three species of Amazona parrots in northeastern Mexico (Doctoral thesis). Texas A \& M University-Kingsville, Texas, USA.

ESRI. (1999). ArcView 3.2. ESRI (Environmental Scientific Research Institute). Redlands, California, USA.

Fielding, A. H. \& Bell, J. F. (1997). A review of methods for the assessment of prediction errors in conservation presence/absence models. Environmental Conservation, 24, 38-49.

Forshaw, J. M. (2006). Parrots of the World: An Identification Guide. Princeton, New Jersey, USA.: Princeton University Press.

Galindo-Leal, C. (1999). La gran región de Calakmul: Prioridades biológicas de conservación y propuesta de modificación de la Reserva de la Biosfera (Reporte Final a World Widlife Fund - México). México D. F.

Gómez-Orduño, J. O. (2006). Disponibilidad y ocupación de cavidades para tres especies de loros del género Amazona spp. en Calakmul, Campeche (Tesis de maestría). El Colegio de la Frontera Sur, Campeche, México.

Gilardi, J. D. \& Munn, C. A. (1998). Patterns of activity, flocking, and habitat use in parrots of the Peruvian Amazon. Condor, 100, 641-653.

Hernández, P. A., Graham, C. H., Master, L. L., \& Albert, D. L. 2006. The effect of sample size and species characteristics on performance of different species distribution modeling methods. Ecography, 29, 773-785.

Herrera-Herrera, J. R. (2001). Spatial and temporal distribution of the avifauna in the wetland and upland habitats of the Calakmul Region, in Mexico (Tesis de doctorado). State University of New York, Syracuse, New York.

Howell, S. N. G. \& Webb, S. (1995). A guide to the birds of Mexico and northern Central America. London, UK: Oxford Univ. Press.

INEGI. (2010). Conjunto de datos vectoriales de la carta de uso del suelo y vegetación: escala 1:250 000. Serie IV (continuo nacional). Aguascalientes, México. Instituto Nacional de Estadística y Geografía.

INEGI. (2014a). Estadísticas a propósito del día internacional de los bosques. Instituto Nacional de Geografía y estadísticas. Recuperado de http://www.inegi.org.mx/ inegi/contenidos/espanol/prensa/contenidos/estadisticas $/ 2014 /$ forestal0.pdf?s=inegi\&c $=2905 \&$ ep $=153$

INEGI. (2014b). Censo Agropecuario 2007, VIII Censo Agrícola, Ganadero y Forestal. Recuperado de 
http://www3.inegi.org.mx/sistemas/sisept/default. aspx? $\mathrm{t}=$ mamb270\&s $=$ est \&c $=32500$

INEGI. (2014c). Encuesta Nacional Agropecuaria 2012. Recuperado de http://www3.inegi.org.mx/sistemas/ sisept $/$ default.aspx $\mathrm{t}=$ mamb327\&s $=$ est $\& \mathrm{c}=33926$

Jetz, W., Wilcove, D. S., \& Dobson, A. P. (2007). Projected impacts of climate and land-use change on the global diversity of birds. PLoS Biology, 5(6), e157.

Juniper, T. \& Parr, M. (1998). Parrots. A guide to parrots of the world. London, UK.: Yale University Press.

Liu, C., Berry, P. M., Dawson, T. P., \& Pearson, R. G. (2005). Selecting thresholds of occurrence in the prediction of species distributions. Ecography, 28, 385-393.

Macías Caballero, C. M. \& Iñigo Elías, E. E. (2003). Evaluación del estado de conservación actual de las poblaciones del loro cabeza amarilla (Amazona oratrix) en México. México, D.F.: Comisión Nacional para el Conocimiento y Uso de la Biodiversidad.

MacKinnon, H. B. 2005. Birds of Yucatan Peninsula. Cancún, México: Amigos de Sian Ka’an, AC.

Marín-Togo, M. C., Monterrubio-Rico, T. C., Renton, K., Rubio-Rocha, Y., Macías-Caballero, C., CancinoMurillo, R., \& Ortega-Rodríguez, J. M. (2012). Reduced current distribution of Psittacidae on the Mexican Pacific coast: potential impacts of habitat loss and capture for trade. Biodiversity and Conservation, 21, 451-473.

Martínez, E. \& Galindo Leal, C. 2002. La vegetación de Calakmul, Campeche, México: clasificación, descripción y distribución. Boletín de la Sociedad Botánica de México, 71, 7-32.

Monterrubio-Rico, T. C., Villaseñor-Gómez, L. E., MarínTogo, M. C., López-Córdova, E. A., Fabian-Turja, B., \& Sorani-Dalbón, V. (2007). Distribución histórica y actual del loro cabeza amarilla (Amazona oratrix) en la costa central del pacífico Mexicano, ventajas y limitaciones en el uso de GARP en especies bajo fuerte presión de tráfico. Ornitología Neotropical, $18,263-276$.

Monterrubio-Rico, T. C., Renton, K., Ortega-Rodríguez, J. M., Pérez-Arteaga, A., \& Cancino-Murillo, R. (2010). The Endangered yellow-headed parrot Amazona oratrix along the Pacific coast of Mexico. Oryx, 44, 602-609.

Morales-Pérez, L. (2005). Evaluación de la abundancia poblacional y recursos alimenticios para tres géneros de psitácidos en hábitats conservados y perturbados de la costa de Jalisco, México (Tesis de maestría). Universidad Nacional Autónoma de México, D. F., México.

Munshi-South, J. \& Wilkinson G. S. (2006). Diet influences life span in parrots (Psittaciformes). The Auk, 123(1), 108-118.
Noss, R., Custi, B., \& Groom, M. J. (2006). Habitat fragmentation. In M. J. Groom, G. K. Meffe, \& C. R. Carroll (Eds.), Principles of Conservation Biology (pp. 213-251). Sunderland: Sinauer Associates, Inc.

Phillips, S. J., Anderson, R. P., \& Schapire, R. E. (2006). Maximum entropy modeling of species geographic distributions. Ecological Modelling, 190, 231-259.

Phillips, S. J. \& Dudík, M. (2008). Modeling of species distribution with MaxEnt: new extensions and a comprehensive evaluation. Ecography, 31, 161-175.

Pizo, M. A. \& Simao, I. (1997). Daily variation in activity and flock size of two parakeet species from Southeastern Brazil. Wilson Bulletin, 109, 343-348.

Plasencia-Vázquez, A. H., Escalona-Segura, G., \& Ligia Guadalupe Esparza-Olguín, L. G. (2014). Modelación de la distribución geográfica potencial de dos especies de psitácidos neotropicales utilizando variables climáticas y topográficas. Acta Zoológica Mexicana (nueva serie), 30(3).

Puc-Cabrera, J. C. (2008). Importancia de las aguadas para los ensambles de aves en el sureste de Campeche (Tesis de licenciatura). Universidad Autónoma de Campeche, Campeche, México.

Redford, K. H. (1992). The empty forest. BioScience, 42, 412-422.

Renton, K. (2002). Amazona oratrix (Ridgway 1887) (Loro cabeza amarilla). In F. A. Noguera, J. H. Vega Rivera, A. N. García Aldrete, \& M. Quesada Avendaño (Eds.), Historia Natural de Chamela (pp. 345-346). México, D. F.: Instituto de Biología, UNAM.

Renton, K. \& Salinas-Melgoza, A. (2004). Climatic variability, nest predation, and reproductive output of lilac-crowned parrots (Amazona finschi) in tropical dry forest of western Mexico. Auk, 121, 1214-1225.

Ridgely, R. S. (1981). The current distribution and status of mainland Neotropical parrots. In R. F. Pasquier (Ed.), Conservation of New World Parrots: Proceedings of the ICBP Parrot Working Group Meeting, St Lucia 1980 (pp. 233-384). Smithsonian Institution Press/ ICBP Technical Publication No 1 .

Ríos-Muñoz, C. A. \& Navarro-Sigüenza, A. G. (2009). Efectos del cambio de uso de suelo en la disponibilidad hipotética de hábitat para los psitácidos de México. Ornitología Neotropical, 20, 491-509.

Rivera-Ortíz, F. A., Oyama, K., Ríos-Muñoz, C. A., Solórzano, S., Navarro-Sigüenza, A. G., \& Arizmendi, M. del C. (2013). Habitat characterization and modeling of the potential distribution of the Military Macaw (Ara militaris) in Mexico. Revista Mexicana de Biodiversidad, 84, 1200-1215.

Romero-Balderas, K. G, Naranjo, E. J., Morales, H. H., \& Nigh, R. B. (2006). Daños ocasionados por vertebrados silvestres al cultivo de maíz en la selva lacandona, Chiapas, México. Interciencia, 31(4), 276-283. 
SEMARNAT. Secretaría de Medio Ambiente y Recursos Naturales. (2010). Norma Oficial Mexicana NOM-059-SEMARNAT-2010. Diario Oficial de la Federación (DOF). Recuperado de http://dof. gob.mx/nota_detalle.php? codigo $=5173091 \& \mathrm{fec}$ ha $=30 / 12 / 2010$

Téllez García, L. (2008). Abundancia relativa y características del hábitat de anidación del loro cabeza amarilla (Amazona oratrix) en diferentes condiciones de conservación de la vegetación (Tesis de maestría). Universidad Michoacana de San Nicólas de Hidalgo, Morelia, Michoacán, México.

Turner II, B. L., Geoghegan, J., \& Foster, D. R. (Eds.). 2004. Integrated land-change science and tropical deforestation in the Southern Yucatan: Final Frontiers. Oxford: Oxford University Press.
Vester, H. F. M., Lawrence, D., Eastman, J. R., Turner II, B. L., Calmé, S., Dickson, R., Pozo, C., \& Sangerman, F. (2007). Land change in the Southern Yucatan and Calakmul Biosphere Reserve: effects on habitat and biodiversity. Ecological Applications, 17, 989-1003.

Villalobos-Zapata, G. J. \& Mendoza Vega, J. (Eds.). (2010). La Biodiversidad en Campeche: Estudio de Estado. Comisión Nacional para el Conocimiento y Uso de la Biodiversidad (CONABIO), Gobierno del Estado de Campeche, Universidad Autónoma de Campeche, El Colegio de la Frontera Sur. México.

Wisz, M. S., Hijmans, R. J., Li, J., Peterson, A. T., Graham C. H., \& Guisan, A. (2008). Effects of sample size on the performance of species distribution models. Diversity and Distributions, 14, 763-773. 